\title{
ON THE AXIALLY-SYMMETRIC STEADY WAVE PROPAGATION IN ELASTIC CIRCULAR RODS*
}

\author{
BY \\ JULIĀN ADEM \\ Instituto de Geofisica, Universidad Nacional de Mexico and \\ Instituto Nacional de la Investigación Cientifica, Mexico, D.F. (Mexico)
}

Summary. In the first part of this report we find an exact solution for the problem of steady wave propagation in an isotropic, elastic circular bar of infinite length, free of stress on its lateral surface and loaded by a harmonic body force, parallel to its axis, whose amplitude is a Dirac delta function. The solution of this problem gives at the same time the solution for a semi-infinite bar with a special prescribed load on its boundary plane.

In the second part, we find a solution for the semi-infinite bar in which the conditions at the boundary plane are prescribed in terms of functions which give implicitly the stresses and displacements.

Finally, using a frequency of interest in current ultrasonic experimental work, we develop a numerical example and compare the result with the case of a low frequency.

1. Infinite bar with the body force $\delta(z) e^{-i \omega t}$. We consider an infinite circular bar of a perfectly elastic isotropic material, free of stresses on the lateral surface and loaded by the body force $\delta(z) e^{-i \omega t}$, where $\delta(z)$ is the Dirac delta function defined by

$$
\begin{gathered}
\delta(z)=0 \quad \text { for } \quad z \neq 0, \\
\int_{-\infty}^{\infty} \delta(z) d z=1,
\end{gathered}
$$

and $\omega$ is a positive number.

The problem is that of determining the displacement at all points of the rod for the steady case (i.e. the time dependence for stresses and displacements is $e^{-i \omega t}$ ).

The general solution. For an elastic isotropic medium the equation of motion is

$$
(\lambda+\mu) \nabla(\nabla \cdot \mathfrak{u})+\mu \nabla^{2} \mathbf{u}+\rho \mathbf{X}=\rho \mathbf{u}^{\prime \prime},
$$

where $\mathfrak{u}$ is the displacement vector; $\mathbf{X}$ is the body force vector, $\lambda$ and $\mu$ elastic constants and $\rho$ the density.

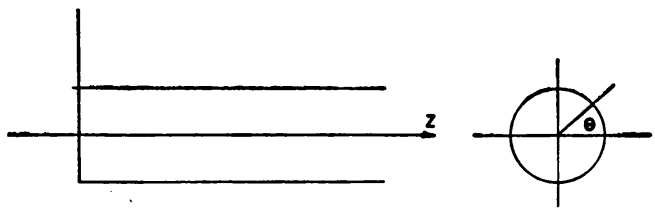

Fig. 1

Using cylindrical coordinates $(r, \theta, z)$ as shown in Fig. 1, we have axial symmetry (i.e. the solution is independent of $\theta$ ).

*Received Aug. 11, 1953; revised manuscript received Dec. 21, 1953. The results presented in this paper were obtained in the course of research sponsored by the Watertown Arsenal under Contract DA-19-020-ORD-2598 when the author was at Brown University, Providence, R. I. 
Let $\mathbf{u}=\nabla \varphi+\nabla \times \mathbf{F}$ and $\mathbf{X}=\mathbf{k} \delta(z) e^{-i \omega t}$, where $\mathbf{k}$ is the unit vector in the direction of the $z$ axis and $\nabla \cdot \mathbf{F}=0$.

Assuming that

$$
\begin{aligned}
& \varphi(r, z, t)=\varphi(r, z) e^{-i \omega t} \\
& \mathbf{F}(r, z, t)=\mathbf{F}(r, z) e^{-i \omega t}
\end{aligned}
$$

and considering that

$$
\nabla[S(z)+C]=\mathbf{k} \delta(z),
$$

where $S(z)$ is the unit step function defined as

$$
\begin{array}{lll}
S(z)=0 & \text { for } & z<0, \\
S(z)=1 & \text { for } & z>0,
\end{array}
$$

and $C$ is an arbitrary constant, we have that (1) is satisfied if

$$
\begin{gathered}
\nabla^{2} \varphi+h^{2} \varphi=-[S(z)+C] \frac{h^{2}}{\omega^{2}}, \\
\nabla^{2}(\nabla \times \mathbf{F})+k^{2}(\nabla \times \mathbf{F})=0,
\end{gathered}
$$

where

$$
h^{2}=\frac{\rho \omega^{2}}{\lambda+2 \mu}, \quad k^{2}=\frac{\rho \omega^{2}}{\mu} .
$$

The formula $d S(z) / d z=\delta(z)$ has been used in the past in a formal way, without rigorous justification. However, recently its validity has been proved on the basis of the theory of Distributions. The reader is referred to L. Schwartz, "Theorie des Distributions".

Using the formula $\nabla^{2}(\nabla \times \mathbf{F})=-\nabla \times \nabla \times(\nabla \times \mathbf{F})$ and assuming that the only component of $\mathbf{F}$ that does not vanish is that in the $\theta$ direction (which will henceforth be called $F$ ), Eq. (3) becomes:

$$
\frac{\partial}{\partial r}\left[\frac{1}{r} \frac{\partial(r F)}{\partial r}\right]+\frac{\partial^{2} F}{\partial z}+k^{2} F=0
$$

while (2) written in cylindrical coordinates becomes (taking $C=0$ ):

$$
\frac{1}{r} \frac{\partial}{\partial r}\left(r \frac{\partial \varphi}{\partial r}\right)+\frac{\partial^{2} \varphi}{\partial z^{2}}+h^{2} \varphi=-\frac{S(z) h^{2}}{\omega^{2}} .
$$

$S(z)$ can be expressed as

$$
S(z)=\frac{1}{2 \pi i} \int_{-\infty}^{\infty} \frac{e^{i \xi z}}{\xi} d \xi,
$$

where the path of integration is the real axis, indented to pass below the origin.

Let

$$
\left.\begin{array}{l}
\varphi(r, z)=\frac{1}{2 \pi} \int_{-\infty}^{\infty} e^{i \xi z} \varphi^{*}(r, \xi) d \xi \\
F(r, z)=\frac{1}{2 \pi} \int_{-\infty}^{\infty} e^{i \xi z} F^{*}(r, \xi) d \xi
\end{array}\right\}
$$


where the path of integration must be such that the solution represents outgoing waves at infinity.

From (4) and (5) we obtain:

$$
\begin{gathered}
\frac{1}{2 \pi} \int_{-\infty}^{\infty}\left[\frac{1}{r} \frac{\partial}{\partial r}\left(r \frac{\partial \varphi^{*}}{\partial r}\right)+\left(h^{2}-\xi^{2}\right) \varphi^{*}+\frac{h^{2}}{i \xi \omega^{2}}\right] e^{i \xi_{z}} d \xi=0, \\
\frac{1}{2 \pi} \int_{-\infty}^{\infty}\left[\frac{\partial}{\partial r} \frac{1}{r} \frac{\partial\left(r F^{*}\right)}{\partial r}+\left(k^{2}-\xi^{2}\right) F^{*}\right] e^{i \xi z} d \xi=0 .
\end{gathered}
$$

These equations are satisfied if

$$
\left.\begin{array}{l}
\frac{1}{r} \frac{\partial}{\partial r}\left(r \frac{\partial \varphi^{*}}{\partial r}\right)+\alpha^{2} \varphi^{*}=-\frac{h^{2}}{i \xi \omega^{2}}, \\
\frac{\partial}{\partial r} \frac{1}{r} \frac{\partial\left(r F^{*}\right)}{\partial r}+\beta^{2} F^{*}=0,
\end{array}\right\}
$$

where $\alpha^{2}=h^{2}-\xi^{2}, \beta^{2}=k^{2}-\xi^{2}$.

A solution of these equations is:

$$
\left.\begin{array}{l}
\varphi^{*}=-\frac{h^{2}}{i \xi \omega^{2} \alpha^{2}}+A(\xi) J_{0}(\alpha r), \\
F^{*}=B(\xi) J_{1}(\beta r),
\end{array}\right\}
$$

where $A(\xi)$ and $B(\xi)$ must be determined from the boundary conditions.

Without loss of generality we assume that the radius of the rod is 1 ; therefore the boundary conditions are $\tau_{r z}=0, \sigma_{r}=0$, at $r=1$.

But

$$
\begin{aligned}
\sigma_{r} & =\lambda\left[\frac{1}{r} \frac{\partial\left(r u_{r}\right)}{\partial r}+\frac{\partial u_{z}}{\partial z}\right]+2 \mu \frac{\partial u_{r}}{\partial r}, \\
\tau_{r z} & =\mu\left[\frac{\partial u_{r}}{\partial z}+\frac{\partial u_{z}}{\partial r}\right]
\end{aligned}
$$

and the displacements in the $r$ and $z$ directions are

$$
\left.\begin{array}{l}
u_{r}=\frac{\partial \varphi}{\partial r}-\frac{\partial F}{\partial z} \\
u_{z}=\frac{\partial \varphi}{\partial z}+\frac{1}{r} \frac{\partial}{\partial r}(r F) .
\end{array}\right\}
$$

Therefore our boundary conditions become

$$
\begin{gathered}
\left\{(\lambda+2 \mu) \frac{\partial^{2} \varphi}{\partial r^{2}}+\lambda \frac{\partial \varphi}{\partial r}+\lambda \frac{\partial^{2} \varphi}{\partial z^{2}}-2 \mu \frac{\partial^{2} F}{\partial z \partial r}\right\}_{r-1}=0 \\
\left\{2 \frac{\partial^{2} \varphi}{\partial r \partial z}-2 \frac{\partial^{2} F}{\partial z^{2}}-k^{2} F\right\}_{r-1}=0
\end{gathered}
$$


and in terms of $\varphi^{*}$ and $F^{*}$,

$$
\left.\begin{array}{r}
{\left[(\lambda+2 \mu) \frac{\partial^{2} \varphi^{*}}{\partial r^{2}}+\lambda \frac{\partial \varphi^{*}}{\partial r}-\lambda \xi^{2} \varphi^{*}-2 i \xi \mu \frac{\partial F^{*}}{\partial r}\right]_{r-1}=0} \\
{\left[2 i \xi \frac{\partial \varphi^{*}}{\partial r}+\left(2 \xi^{2}-k^{2}\right) F^{*}\right]_{r-1}=0}
\end{array}\right\}
$$

Substituting (8) in (10)

$$
\begin{gathered}
A(\xi) 2 i \xi \alpha J_{1}(\alpha)+B(\xi)\left(k^{2}-2 \xi^{2}\right) J_{1}(\beta)=0, \\
A(\xi)\left\{2 \mu \alpha J_{1}(\alpha)-\left[(\lambda+2 \mu) \alpha^{2}+\lambda \xi^{2}\right] J_{0}(\alpha)\right\}+B(\xi)\left[-2 i \mu \beta \xi J_{0}(\beta)+2 i \xi \mu J_{1}(\beta)\right]=-\frac{\lambda \xi h^{2}}{i \omega^{2} \alpha^{2}} .
\end{gathered}
$$

The solution of this system of equations is

$$
\left.\begin{array}{l}
B(\xi)=-\frac{\lambda h^{2} \xi^{2} J_{1}(\alpha)}{2 \mu \omega^{2} \alpha \Delta} \\
A(\xi)=-\frac{i \lambda h^{2}\left(\frac{1}{2} k^{2}-\xi^{2}\right) \xi J_{1}(\beta)}{2 \mu \omega^{2} \alpha^{2} \Delta}
\end{array}\right\}
$$

where $\Delta=\xi^{2} \alpha \beta J_{1}(\alpha) J_{0}(\beta)-\alpha \frac{1}{2} k^{2} J_{1}(\alpha) J_{1}(\beta)+\left(\frac{1}{2} k^{2}-\xi^{2}\right)^{2} J_{0}(\alpha) J_{1}(\beta)$. Substituting (11) into (8), and the resultant values of (8) into (6), we obtain

$$
\left.\begin{array}{l}
\varphi(r, z)=-\frac{h^{2}}{2 \pi i \omega^{2}} \int_{-\infty}^{\infty} \frac{e^{i \xi z}}{\xi \alpha^{2}} d \xi-\frac{i \lambda h^{2}}{4 \mu \omega^{2} \pi} \int_{-\infty}^{\infty} \frac{\xi\left(-\xi^{2}+\frac{1}{2} k^{2}\right) J_{1}(\beta) J_{0}(\alpha r) e^{i \xi z}}{\alpha^{2} \Delta} d \xi, \\
F(r, z)=-\frac{\lambda h^{2}}{4 \mu \omega^{2} \pi} \int_{-\infty}^{\infty} \frac{\xi^{2} J_{1}(\alpha) J_{1}(\beta r) e^{i \xi z}}{\alpha \Delta} d \xi .
\end{array}\right\}
$$

In order to compute the integrals that appear in this solution it is convenient to determine the roots of $\Delta / \beta=0$.

The frequency equation. The equation

$$
\frac{\Delta}{\beta} \equiv \xi^{2} \alpha J_{1}(\alpha) J_{0}(\beta)-\frac{\alpha}{\beta} \frac{1}{2} k^{2} J_{1}(\alpha) J_{1}(\beta)+\left(\frac{1}{2} k^{2}-\xi^{2}\right)^{2} J_{0}(\alpha) \frac{J_{1}(\beta)}{\beta}=0,
$$

where $\alpha=\left(h^{2}-\xi^{2}\right)^{1 / 2}, \beta=\left(k^{2}-\xi^{2}\right)^{1 / 2}$, known as Pochhammer's frequency equation, has been the subject of several papers*, because it gives the modes of longitudinal wave propagation in an infinite circular rod, having stress free surface (velocity of the wave $=$ $\omega / \xi$; wave length $=2 \pi / \xi$ ).

In this equation, given the elastic constants, $\xi$ is a multiple-valued function of the angular frequency $\omega$. For the purpose of computing our integrals we are interested in determining all the roots of Eq. (13), corresponding to the given frequency $\omega$.

Let us consider the frequency equation (13) for large $|\xi|$. In this case $\alpha$ and $\beta$ can be written as

$$
\begin{aligned}
& \alpha \cong i\left(\xi-h^{2} / 2 \xi\right), \\
& \beta \cong i\left(\xi-k^{2} / 2 \xi\right)
\end{aligned}
$$

*D. Bancroft, Phys. Rev., 59, 588 (1941); A. H. Holden, Bell System Technical J., 30, No. 4 (1951); R. M. Davies, Trans. Roy. Soc., (London), A, No. 821, 240 (1948). See also the references mentioned in these papers. 
and the frequency equation for sufficiently large $|\xi|$ becomes

$$
2 i \xi-\cosh 2 \xi=0 \text {. }
$$

Let $\xi=\xi_{1}+i \xi_{2}$, where $\xi_{1}$ and $\xi_{2}$ are real; then Eq. (14) can be written as the pair

$$
\begin{array}{r}
2 \xi_{2}+\cos 2 \xi_{2} \cosh 2 \xi_{1}=0, \\
-2 \xi_{1}+\sin 2 \xi_{2} \sinh 2 \xi_{1}=0 .
\end{array}
$$

Considering that $|\xi|$ is large, these equations have solution only if $\left|\xi_{1}\right|$ is large and, since

$$
\xi_{1} / \sinh \xi_{1} \rightarrow 0 \quad \text { when } \quad\left|\xi_{1}\right| \rightarrow \infty,
$$

it can easily be shown that there is an infinite number of roots of Eq. (14) which are approximately at the points

$$
\begin{aligned}
& \xi= \pm \frac{1}{2} \log 2 k \pi-i k \pi / 2, \\
& \xi= \pm \frac{1}{2} \log 2 p \pi+i p \pi / 2,
\end{aligned}
$$

where $k$ is a large positive even integer and $p$ is a large positive odd integer.

In Eq. (13) the variable is $\xi^{2}$; therefore we must consider the solution for $\xi$ as well as for $-\xi$. For the minus sign case $\alpha$ and $\beta$ are given by

$$
\alpha \cong-i \xi\left(1-\frac{h^{2}}{2 \xi^{2}}\right), \quad \beta \cong-i \xi\left(1-\frac{k^{2}}{2 \xi^{2}}\right)
$$

and the frequency equation becomes*

$$
2 i \xi+\cosh 2 \xi=0,
$$

whose roots are those of (14) with opposite sign.

Therefore the frequency equation (13) for large $|\xi|$ can be written as

or

$$
(2 i \xi-\cosh 2 \xi)(2 i \xi+\cosh 2 \xi)=0,
$$

$$
4 \xi^{2}+\cosh ^{2} 2 \xi=0
$$

and there are an infinite number of roots of it which are approximately at the points

$$
\xi= \pm \frac{1}{2} \log 2 k \pi \pm i \frac{k \pi}{2}
$$

where $k$ is a large positive integer and where all combinations of sign are allowed.

Since there are no roots of (13) on the axes for large $|\xi|$ it follows that there are a finite number of roots on them.

Finally, we are going to show that there are no roots in the neighborhood of the real axis (except at the axis itself). Let us denote (13) by the brief notation

$$
F(\xi)=0 ;
$$

this equation can be written as

$$
F\left(\xi_{1}\right)+i \xi_{2} F^{\prime}\left(\xi_{1}\right)+\cdots=0
$$

${ }^{*}$ As was also shown by C. W. Curtis in a private letter to the author. 
and, if $\xi_{2}$ is small enough to neglect $\xi_{2}^{2}$, since $F^{\prime}\left(\xi_{1}\right) \neq 0$ when $F\left(\xi_{1}\right)=0$, and $F\left(\xi_{1}\right)$ and $F^{\prime}\left(\xi_{1}\right)$ are both real, it follows that $\left(13^{\prime \prime}\right)$ can be satisfied only if $\xi_{2}=0$.

Evaluation of the integrals. From now on we shall consider the solution for $z>0$.

The integral

$$
I_{1}=\int_{-\infty}^{\infty} \frac{e^{i \xi z}}{\xi \alpha^{2}} d \xi
$$

can easily be evaluated by using Cauchy's residue theorem.* There are three poles on the real axis: one at $\xi=0$, and the other two at $\xi= \pm h$; in the rest of the complex plane the integrand is regular. To satisfy the condition at infinity (outgoing waves) we choose the path of integration as shown in Fig. 2, i.e. from $\xi=-R$ to $\xi=R$ indented at $\xi= \pm h$ and $\xi=0$ to pass above the real axis for $\xi=-h$ and below it for the other poles; and then from $\xi=R$ to $\xi=-R$ along a semicircle $\Gamma$ with center in the origin. Integrating on the chosen path and letting $R \rightarrow \infty$, since (by Jordan's lemma) the integral on the semicircle $\Gamma$ goes to zero we obtain:

$$
\int_{-\infty}^{\infty} \frac{e^{i \xi z}}{\xi \alpha^{2}} d \xi=\frac{\pi i}{h^{2}}\left[2-e^{i h z}\right]
$$

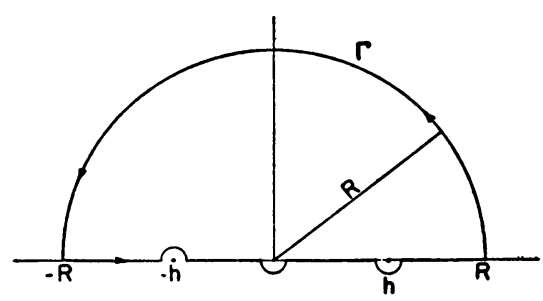

FIG. 2

Now let us consider the integral

$$
I_{2}=\int_{-\infty}^{\infty} \frac{\xi\left(-\xi^{2}+\frac{1}{2} k^{2}\right) J_{1}(\beta) J_{0}(\alpha r) e^{i \xi z}}{\alpha^{2} \Delta} d \xi ;
$$

dividing the numerator and denominator of the integrand by $\beta$ we obtain:

$$
I_{2}=\int_{-\infty}^{\infty} f(\xi) J_{0}(\alpha r) e^{i \xi z} d \xi
$$

where

$$
f(\xi)=\frac{\xi\left(-\xi^{2}+\frac{1}{2} k^{2}\right) \frac{J_{1}(\beta)}{\beta}}{\alpha^{2} \Delta / \beta} .
$$

The numerator and the denominator of the integrand are both regular functions and the only singularities of the integrand in the finite plane are simple poles corresponding to the roots of the equation $\alpha^{2} \Delta / \beta=0$.

"See E. T. Whittaker and G. N. Watson, "A course of modern analysis", Chapter VI; or any of the standard books on complex variable. 
Let $\xi_{1}, \xi_{2}, \cdots, \xi_{n}$ be the positive real roots and $\xi_{n+1}, \xi_{n+2}, \cdots$ the complex roots of $\Delta / \beta=0$.

We shall again use Cauchy's residue theorem, choosing the path of integration as shown in Fig. 3.

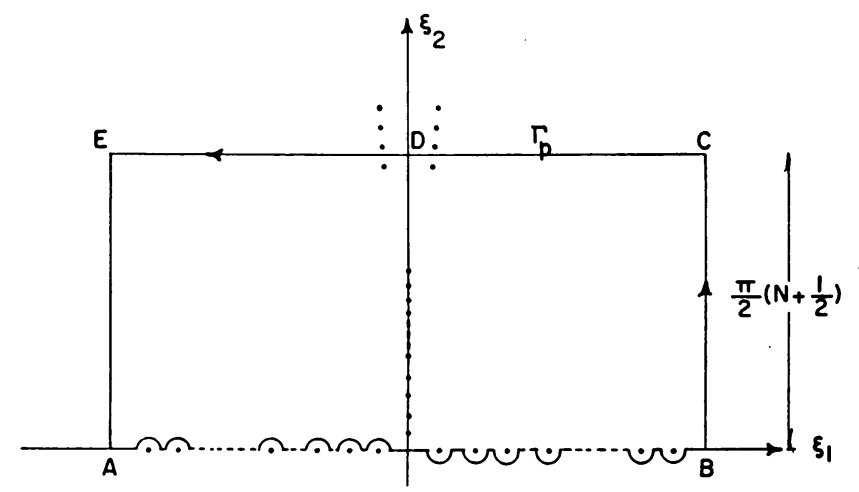

Fra. 3

The path goes from $A$ to $B$ on the real axis, indenting it at the poles to pass above the real axis for negative poles and below the axis for positive ones (the reason for this choice of the path is to satisfy the condition of "outgoing waves" at infinity); then we close the contour of integration by a family of paths $\Gamma_{p}$ such that

$$
\lim _{|\xi| \rightarrow \infty} \int_{\Gamma_{P}} f(\xi) J_{0}(\alpha r) e^{i \xi z} d \xi=0 .
$$

In our case we have used the family of rectangles shown in Fig. 3: the side $C E$ passes between two consecutive pairs of roots of the frequency equation (for large $|\xi|$ ) and $C D=D E=E A=\frac{1}{2} \pi\left(N+\frac{1}{2}\right)$, where $N$ is a large positive integer.

Integrating on the chosen path and letting $N \rightarrow \infty$ we obtain:

$$
\int_{-\infty}^{\infty} f(\xi) J_{0}(\alpha r) e^{i \xi z} d \xi=2 \pi i \sum_{a=1}^{\infty} R_{q}-\lim _{N \rightarrow \infty} \int_{\Gamma_{P}} f(\xi) J_{0}(\alpha r) e^{i \xi z} d \xi,
$$

where $R_{a}$ are the residues at the poles on the positive real axis and upper half-plane.

But, since

$$
\lim _{N \rightarrow \infty} \int_{\Gamma_{P}} f(\xi) J_{0}(\alpha r) e^{i \xi z} d \xi=0,
$$

evaluating the residues we obtain:

$$
\int_{-\infty}^{\infty} f(\xi) J_{0}(\alpha r) e^{i \xi z} d \xi=2 \pi i \sum_{\alpha=1}^{\infty} g\left(\xi_{\alpha}\right) J_{0}\left(\alpha_{\alpha} r\right) e^{i \xi \varepsilon^{z}}+\frac{\pi i}{h^{2}-\frac{1}{2} k^{2}} e^{i h z},
$$

where

$$
g(\xi)=\frac{\xi\left(\frac{1}{2} k^{2}-\xi^{2}\right) J_{1}(\beta)}{\alpha^{2} \beta \frac{d}{d \xi}(\Delta / \beta)}
$$

and $\alpha_{a}=[\alpha]_{\xi=\xi_{a}}$. 
In a similar way the last integral of (12) can be evaluated, and we obtain:

$$
\int_{-\infty}^{\infty} \frac{\xi J_{1}(\alpha) J_{1}(\beta r) e^{i \xi_{2}}}{\alpha \Delta} d \xi=2 \pi i \sum_{a=1}^{\infty} t\left(\xi_{a}\right) J_{1}\left(\beta_{a} r\right) e^{i \xi_{\alpha} z},
$$

where

$$
t(\xi)=\frac{\xi^{2} J_{1}(\alpha)}{\alpha \beta \frac{d}{d \xi}(\Delta / \beta)}
$$

and

$$
\beta_{a}=[\beta]_{\xi-\xi_{a}} .
$$

Substituting (15), (16) and (17) in (12) we see that the terms in $e^{\text {ins }}$ cancel and obtain:

$$
\left.\begin{array}{l}
\varphi(r, z)=-\frac{1}{\omega^{2}}+\frac{\lambda h^{2}}{2 \mu \omega^{2}} \sum_{a=1}^{\infty} g\left(\xi_{a}\right) J_{0}\left(\alpha_{a} r\right) e^{i \xi_{a} s}, \\
F(r, z)=-\frac{\lambda h^{2} i}{2 \pi \omega^{2}} \sum_{a=1}^{\infty} t\left(\xi_{a}\right) J_{1}\left(\beta_{a} r\right) e^{i \xi_{a} z} .
\end{array}\right\}
$$

Substituting (18) in (9) and considering the time dependence, we obtain

$$
\begin{aligned}
& u_{z}(r, z, t)=i \sum_{a=1}^{\infty}\left\{\xi_{a} G\left(\xi_{a}\right) J_{0}\left(\alpha_{a} r\right)-\beta_{a} T\left(\xi_{a}\right) J_{0}\left(\beta_{a} r\right)\right\} e^{i\left(\xi_{a}-\omega t\right)}, \\
& u_{r}(r, z, t)=\sum_{a=1}^{\infty}\left\{-\alpha_{a} G\left(\xi_{a}\right) J_{1}\left(\alpha_{a} r\right)-\xi_{a} T\left(\xi_{a}\right) J_{1}\left(\beta_{a} r\right)\right\} e^{i\left(\xi_{a} z-\omega t\right)},
\end{aligned}
$$

where

$$
\left.\begin{array}{l}
G(\xi)=\frac{\lambda h^{2}}{2 \mu \omega^{2}} \frac{\xi\left(-\xi^{2}+\frac{1}{2} k^{2}\right) J_{1}(\beta)}{\alpha^{2} \beta \frac{d}{d \xi}(\Delta / \beta)}, \\
T(\xi)=\frac{\lambda h^{2}}{2 \mu \omega^{2}} \frac{\xi^{2} J_{1}(\alpha)}{\alpha \beta \frac{d}{d \xi}(\Delta / \beta)},
\end{array}\right\}
$$

$\xi_{1}, \xi_{2}, \cdots, \xi_{n}$ are the roots on the positive real axis of $\Delta / \beta=0 ; \xi_{n+1}, \xi_{n+2}, \cdots$ are the roots in the upper half plane;

$$
\begin{aligned}
\alpha & =\left(h^{2}-\xi^{2}\right)^{1 / 2}, & \beta & =\left(k^{2}-\xi^{2}\right)^{1 / 2}, \\
h & =\left(\frac{\rho}{\lambda+2 \mu}\right)^{1 / 2} \omega, & k & =\left(\frac{\rho}{\mu}\right)^{1 / 2} \omega, \\
\alpha_{a} & =[\alpha]_{\xi-\xi_{a}}, & \beta_{a} & =[\beta]_{\xi-\xi_{a}} .
\end{aligned}
$$

In the solution (19), (20) the terms due to the complex roots have the decay factor $e^{-a s}$ (" $a$ " real and positive) and for $z$ sufficiently large, they become very small and can be neglected. As we consider the solution for smaller $z$, some of those terms probably must be considered (those that correspond to the poles that are closest to the real axis). 
We will obtain better information about the characteristics of the solution (19), (20) in the last section where we discuss a numerical example.

To obtain the solution for $z<0$ we integrate as in Fig. 3, following the same path $A B$, but instead of the path $B C D E A$ we take its reflection in the real axis. It can easily be checked that $u_{z}(r, z)=u_{z}(r,-z), u_{r}(r, z)=-u_{z}(r,-z)$, as is to be expected by considerations of symmetry.

2. Semi-infinite bar. Consider the same bar as in the previous problem. The equation (1) without body force is

$$
(\lambda+\mu) \nabla(\nabla \cdot \mathfrak{u})+\mu \nabla^{2} \mathbf{u}=\rho \mathbf{u}^{\prime \prime} .
$$

We look for a steady axially-symmetric solution of Eq. (22), which satisfies the stress-free condition at the lateral surface of the bar and at the plane $z=0$ is of the type $u_{z}=u_{s}(r) e^{-i \omega t}, u_{r}=u_{r}(r) e^{-i \omega t}$ [or $\sigma_{z}=\sigma_{z}(r) e^{-i \omega t}, \tau_{r z}=\tau_{r s}(r) e^{-i \omega t}$ ].

The solution (19), (20) of the above problem suggests that we assume:

$$
\mathbf{u}=\nabla \boldsymbol{\varphi}+\nabla \times \mathbf{F} ; \quad \nabla \cdot \mathbf{F}=\mathbf{0},
$$

where

$$
\begin{aligned}
& \varphi(r, z, t)=\varphi(r, \xi) e^{i(\xi z-\omega t),} \\
& \mathbf{F}(r, z, t)=\mathbf{F}(r, \xi) e^{i(\xi z-\omega t),}
\end{aligned}
$$

where $\xi$ is to be determined from boundary conditions. Substituting (23) in (22) we obtain

$$
\begin{aligned}
& \frac{1}{r} \frac{\partial}{\partial r}\left(r \frac{\partial \varphi}{\partial r}\right)+\alpha^{2} \varphi=0 \\
& \frac{\partial}{\partial r} \frac{1}{r} \frac{\partial(r F)}{\partial r}+\beta^{2} F=0
\end{aligned}
$$

where $F$ is the $\theta$ component of the vector $\mathrm{F}$, the other components being zero; and $\alpha$ and $\beta$ have the same meaning as before. Therefore

$$
\begin{aligned}
& \varphi(r, \xi)=A(\xi) J_{0}(\alpha r), \\
& F(r, \xi)=B(\xi) J_{1}(\beta r) .
\end{aligned}
$$

From the boundary conditions at $r=1$

$$
A(\xi)=-B(\xi) \frac{\left(\frac{1}{2} k^{2}-\xi^{2}\right) J_{1}(\beta)}{i \xi \alpha J_{1}(\alpha)},
$$

where $\xi$ must be a root of the frequency equation (13).

From (9) we obtain

$$
\left.\begin{array}{l}
u_{z}=\sum_{a=1}^{\infty} B\left(\xi_{a}\right)\left[-\xi_{a} M\left(\xi_{a}\right) J_{0}\left(\alpha_{a} r\right)+\beta_{a} J_{0}\left(\beta_{a} r\right)\right] e^{i\left(\xi_{a} s-\omega t\right)}, \\
u_{r}=i \sum_{a=1}^{\infty} B\left(\xi_{a}\right)\left[-\alpha_{a} M\left(\xi_{a}\right) J_{1}\left(\alpha_{a} r\right)-\xi_{a} J_{1}\left(\beta_{a} r\right)\right] e^{i\left(\xi_{a}-\omega t\right)},
\end{array}\right\}
$$


where

$$
M\left(\xi_{a}\right)=\frac{\left(\frac{1}{2} k^{2}-\xi_{a}^{2}\right) \cdot J_{1}\left(\beta_{q}\right)}{\xi_{q} \alpha_{q} J_{1}\left(\alpha_{q}\right)}
$$

and $\xi_{a}, \alpha_{a}, \beta_{a}$ have the same meaning as in solution (19), (20).

Similarly we can express $\sigma_{z}$ and $\tau_{r_{z}}$ in terms of the arbitrary functions $B\left(\xi_{q}\right)$.

In order to satisfy the conditions at infinity (outgoing waves of finite amplitude), in the solution (24) we must include only the terms corresponding to the roots of the frequency equation that are on the positive real axis or above the real axis.

At the plane $z=0$ we can prescribe displacements or stresses by prescribing the functions $B\left(\xi_{a}\right)$. To do this we need to know the roots, $\xi_{a}$, of the frequency equation (13). If we know only some of the roots, then for the other roots we can take $B\left(\xi_{a}\right)=0$. We can in this way construct solutions in which at $z=0, u_{z}$ and $u_{r}$ are given by (24); (or $\sigma_{z}$ and $\tau_{r z}$ by the corresponding formulas).

The problem of an infinite bar with the body force $\delta(z) e^{-i \omega t}$, where $\delta(z)$ is the Dirac delta function, corresponds to the case in which $B\left(\xi_{i}\right)=-i T\left(\xi_{i}\right)$, where $T\left(\xi_{i}\right)$ is given by (21).

3. Numerical example. Let us consider a bar of radius equal to $1 \mathrm{~cm}$.

We shall take Poisson's ratio equal to $1 / 3: \nu=1 / 3$. For this case* $V_{l}=2 V_{l}$, where $V_{l}=(\lambda+2 \mu / \rho)^{1 / 2}$ and $V_{t}=(\mu / \rho)^{1 / 2}$ are the velocities of the longitudinal (compressional) and transversal (shear) waves in an infinite medium.

Therefore $k=2 h$.

Let

$$
\xi=h y,
$$

then the frequency equation (13) becomes:

$$
\begin{aligned}
y^{2}\left(1-y^{2}\right)^{1 / 2} J_{1}\left[h\left(1-y^{2}\right)^{1 / 2}\right] J_{0} & {\left[h\left(4-y^{2}\right)^{1 / 2}\right] } \\
& -\frac{2\left(1-y^{2}\right)^{1 / 2}}{h\left(4-y^{2}\right)^{1 / 2}} J_{1}\left[h\left(1-y^{2}\right)^{1 / 2}\right] J_{1}\left[h\left(4-y^{2}\right)^{1 / 2}\right] \\
+ & \frac{\left(2-y^{2}\right)^{2}}{\left(4-y^{2}\right)^{1 / 2}} J_{0}\left[h\left(1-y^{2}\right)^{1 / 2}\right] J_{1}\left[h\left(4-y^{2}\right)^{1 / 2}\right]=0 .
\end{aligned}
$$

For the moment we are interested in the case of frequencies, $\omega / 2 \pi$, in the ultrasonic range which vary approximately from $5 \times 10^{6}$ cycles/sec. to $25 \times 10^{6}$ cycles $/ \mathrm{sec}$. To be specific we are going to consider the case $\omega=2 \pi \times 10^{7}$; furthermore we shall take

$$
V_{l}=\left(\frac{\lambda+2 \mu}{\rho}\right)^{1 / 2}=5 \times 10^{5} \mathrm{~cm} / \mathrm{sec} .
$$

then

$$
h=\frac{\omega}{V_{l}}=40 \pi .
$$

${ }^{*}$ L. Gold, Brown University Techn. Report WA-6487/4. 
The positive real roots of the frequency equation (26) are in the interval $0<y<2.16$. There are approximately 130 positive real roots. Those in the interval $.85<y<2.16$ are given in Table 1 .

TABLE 1

Roots of the frequency equation (26) for $h=40 \pi$ in interval: $0.85>y>2.16$.

\begin{tabular}{llll}
\hline \hline & & & \\
.8514 & 1.0006 & 1.6414 & 1.9263 \\
.8609 & 1.0336 & 1.6592 & 1.9332 \\
.8715 & 1.0733 & 1.6753 & 1.9397 \\
.8835 & 1.1090 & 1.6914 & 1.9459 \\
.8949 & 1.1437 & 1.707 & 1.9517 \\
& & & \\
.9046 & 1.1775 & 1.7222 & 1.9572 \\
.9126 & 1.2119 & 1.7368 & 1.9624 \\
.9208 & 1.2418 & 1.7509 & 1.9672 \\
.9291 & 1.2725 & 1.7646 & 1.9717 \\
.9387 & 1.3018 & 1.7778 & 1.9758 \\
& & & \\
.9466 & 1.3257 & 1.7905 & 1.9796 \\
.9534 & 1.3571 & 1.8030 & 1.9843 \\
.9589 & 1.3846 & 1.8147 & 1.9862 \\
.9645 & 1.4099 & 1.8262 & 1.9893 \\
.9708 & 1.4344 & 1.8373 & 1.9915 \\
& & & \\
.9759 & 1.4583 & 1.8479 & 1.9937 \\
.9810 & 1.4817 & 1.8581 & 1.9956 \\
.9855 & 1.5038 & 1.8680 & 1.9971 \\
.9894 & 1.5251 & 1.8774 & 1.9983 \\
.9926 & 1.5464 & 1.8865 & 1.9992 \\
& & & \\
.9954 & 1.5664 & 1.8952 & 1.9997 \\
.9974 & 1.5863 & 1.9035 & 2.1500 \\
.9989 & 1.6056 & 1.9115 & \\
.9998 & 1.6236 & 1.9191 & \\
\hline & & & \\
\hline
\end{tabular}

The roots on the imaginary axis closest to the real axis are at

$$
\begin{aligned}
& y=.159 i, \\
& y=.192 i .
\end{aligned}
$$

The roots $\xi_{a}$ are $40 \pi$ times the corresponding roots of (26). Substituting them in the solution (19-20), we see that of the terms corresponding to real roots the ones with largest amplitudes are those that correspond to the roots near $y=1$. If in the solution we consider only the terms corresponding to the roots in $.86<y<1.4$, we get a result with an accuracy roughly speaking, of $1 \%$.

The terms corresponding to the imaginary roots have the decay factor

$$
e^{-40 \pi\left|y_{0}\right| z}
$$


where the smallest value of $\left|y_{a}\right|$ is 0.159 , and therefore such terms can be neglected in the solution, except in the neighborhood of $z=0$. If we take $z>1 / 2$, for example, we can ignore such terms.

The solution, considering only the roots in the interval $.86 \leq y<1.4$, is

$u_{z}(r, z, t)=i \frac{1}{40 \pi V_{l}^{2}} \sum_{a}\left[T_{a} J_{0}\left(\alpha_{a} r\right)+S_{a} J_{0}\left(\beta_{a} r\right)\right] e^{i\left(40 x y_{a} z-\omega t\right)}$

$$
\left.+\sum_{p}\left[R_{p} \frac{I_{0}\left(\alpha_{p} r\right)}{I_{0}\left(\alpha_{p}\right)}+S_{p} J_{0}\left(\beta_{p} r\right)\right] e^{i\left(40 r y_{p} z-\omega t\right)},\right\}
$$

$u_{r}(r, z, t)=\frac{1}{40 \pi V_{l}^{2}} \sum_{a} M_{a} J_{1}\left(\beta_{a} r\right) e^{i\left(40 \pi y_{a} s-\omega \ell\right)}$

where $y_{q}, \alpha_{q}, \beta_{q}, T_{q}, S_{q}, y_{p}, \alpha_{p}, \beta_{p}, R_{p}, S_{p}, M_{q}$ are given in Table 2.

\section{TABLE 2}

\begin{tabular}{|c|c|c|c|c|c|}
\hline$y_{a}$ & $\alpha_{q}$ & $\beta_{q}$ & $T_{q}$ & $S_{q}$ & $M_{q}$ \\
\hline .8609 & 63.9127 & 226.8361 & +.02 & +.05 & \\
\hline $.8715^{\circ}$ & 61.7387 & 226.2329 & -.09 & +.28 & \\
\hline .8835 & 58.7479 & 225.4412 & +.11 & +.15 & \\
\hline .8949 & 56.0587 & 224.7626 & -.10 & +.17 & \\
\hline .9046 & 53.4575 & 224.1217 & +.05 & +.17 & \\
\hline .9126 & 51.8238 & 223.7448 & +.02 & -.22 & \\
\hline .9209 & 48.9587 & 223.0913 & -.11 & -.16 & \\
\hline .9291 & 46.5082 & 222.5761 & +.14 & -.18 & \\
\hline .9387 & 43.5677 & 221.9729 & -.15 & -.16 & \\
\hline .9466 & 40.7403 & 221.4325 & +.13 & -.18 & \\
\hline .9534 & 38.0762 & 220.9676 & -.08 & -.13 & \\
\hline .9581 & 36.0279 & 220.6032 & -.03 & +.28 & \\
\hline .9645 & 33.4141 & 220.2010 & +.15 & +.31 & \\
\hline .9708 & 31.039 & 219.8617 & -.27 & +.35 & \\
\hline .9759 & 27.3571 & 219.3716 & +.21 & +.22 & \\
\hline .9810 & 24.3914 & 219.0198 & -.23 & +.23 & \\
\hline .9855 & 21.4760 & 218.7056 & +.25 & +.24 & \\
\hline .9894 & 18.2590 & 218.4166 & -.27 & +.30 & \\
\hline .9926 & 15.2305 & 218.1778 & +.30 & +.38 & .20 \\
\hline .9954 & 12.3151 & 217.9893 & -.32 & +.46 & .26 \\
\hline .9974 & 9.2363 & 217.8385 & -.45 & +.80 & .44 \\
\hline .9989 & 6.1575 & 217.7380 & -.45 & +.94 & .54 \\
\hline .9998 & 5.0266 & 217.6752 & +.77 & +2.06 & 1.18 \\
\hline
\end{tabular}




\begin{tabular}{|c|c|c|c|c|c|c|}
\hline$y_{p}$ & $\alpha_{p}$ & $\beta_{p}$ & $R_{p}$ & $S_{p}$ & $M_{q}$ & \\
\hline 1.0006 & 4.3480 & 217.6123 & +.42 & -7.31 & -4.07 & _ \\
\hline 1.0336 & 34.2937 & 214.9357 & +.08 & +1.55 & .92 & \\
\hline 1.0733 & 48.8205 & 212.1083 & +.06 & -1.20 & -.73 & \\
\hline 1.1090 & 60.2308 & 209.1552 & +.03 & +.79 & .54 & \\
\hline 1.1437 & 69.7184 & 206.1769 & 0 & -.61 & -.36 & \\
\hline 1.1775 & 78.1253 & 203.1484 & 0 & +.47 & .36 & \\
\hline 1.2119 & 86.4945 & 199.7304 & 0 & -.21 & -.18 & \\
\hline 1.2418 & 92.5641 & 196.9909 & 0 & +.31 & & \\
\hline 1.2725 & 98.7718 & 193.9490 & 0 & -.29 & & \\
\hline 1.3018 & 104.7786 & 191.1978 & 0 & +.22 & & \\
\hline 1.3257 & 110.2827 & 187.6415 & & -.11 & & \\
\hline 1.3571 & 115.2716 & 184.6255 & & +.19 & & \\
\hline 1.3846 & 120.5997 & 181.1949 & & -.11 & & \\
\hline 1.4099 & 125.0859 & 178.1162 & & +.11 & & \\
\hline 1.4344 & 129.3334 & 175.0500 & & -.11 & & \\
\hline 1.4583 & 133.3295 & 172.034 & & +.11 & & \\
\hline 1.4817 & 137.6146 & 168.616 & & -.11 & & \\
\hline 1.5038 & 141.1706 & 165.6629 & & +.11 & & \\
\hline 1.5251 & 145.0163 & 162.3076 & & -.07 & & \\
\hline
\end{tabular}

Some remarks. The longitudinal displacement for $z$ not near the origin (say, $z>1 / 2$ ) consists of approximately 130 harmonic waves (one for each positive real root of the frequency equation) which travel with velocities varying from $V_{l} / .118$ to $V_{l} / 2.15$ and with wave length varying from $(1 / 20)(1 / 0.118)$ to $(1 / 20)(1 / 2.15)$, respectively. Since in the solution (27), $J_{0}\left(\alpha_{a} r\right), J_{0}\left(\beta_{a} r\right)$ and $I_{0}\left(\alpha_{a} r\right) / I_{0}\left(\alpha_{a}\right)$ have absolute value less than 1 , the coefficients $T_{a}, S_{q}, R_{p}, S_{p}, M_{q}$ give the order of magnitude of the amplitude of the different waves. The waves of largest amplitude are those which travel with a velocity approximately equal to $V_{\imath}=[(\lambda+2 \mu) / \rho]^{1 / 2}$ and with wave length approximately $1 / 20$; the closer the velocity is to $V_{l}$ the larger the amplitude of the wave is; and it is interesting to point out that the amplitude of the wave that travels with the velocity $V_{l} / 1.0006$ is remarkably larger than the others. Therefore if we are looking for an approximate solution, it is sufficient to consider only the roots near the point $\xi=h($ i.e. $y=1)$.

An example with low frequency. Now let us consider the solution for small values of $\omega$.

When $\omega \rightarrow 0, h \rightarrow 0, k \rightarrow 0$ and the frequency equation (26) has only one root at $y^{2}=3 / 2$ and therefore the solution will have only one term.

The wave velocity

$$
V \rightarrow\left(\frac{E}{\rho}\right)^{1 / 2}=\left(\frac{2}{3}\right)^{1 / 2} V_{l}
$$

and the wave length tends to infinity.

Let us consider $h=3\left(\omega=3 V_{l}\right)$. In this case the frequency equation has 3 positive real roots which are at $y=.692, y=1.080, y=2.105$ and the solution (not near the origin) [considering the imaginary part of (19), (20) only] is: 


$$
\begin{aligned}
u_{z}=\frac{1}{3} V_{l}^{-2}[.397 & \left.J_{0}(2.1651 r)+.574 J_{0}(5.6286 r)\right] \cos \left(2.076 z-3 V_{l} t\right) \\
& +\frac{1}{3} V_{l}^{-2}\left[-.004 I_{0}(1.8792 r)-0.604 J_{0}(3.8043 r)\right] \cos \left(3.540 z-3 V_{l} t\right) \\
& +\frac{1}{3} V_{l}^{-2}\left[-.008 I_{0}(5.5569 r)+0.011 I_{0}(1.9695 r)\right] \cos \left(6.315 z-3 V_{l} t\right), \\
u_{r}=\frac{1}{3} V_{l}^{-2}[-0.406 & \left.J_{1}(2.1657 r)+0.021 J_{1}(5.6286 r)\right] \sin \left(2.076 z-3 V_{l} t\right) \\
& +\frac{1}{3} V_{l}^{-2}\left[-0.002 I_{1}(1.8792 r)-0.562 J_{1}(3.8043 r)\right] \sin \left(3.540 z-3 V_{l} t\right) \\
& +\frac{1}{3} V_{l}^{-2}\left[-0.007 I_{1}(5.5669 r)+0.036 I_{1}(1.9695 r)\right] \sin \left(6.315 z-3 V_{l} t\right) .
\end{aligned}
$$

In this solution the amplitude of the two first terms decreases as $r$ increases, while that of the last one increases and gives practically the only contribution near the surface of the rod. The velocity with which the wave corresponding to the last term travels is almost that of Rayleigh surface wave.

The wave lengths are

$$
L_{1}=\frac{2 \pi}{2.076}, \quad L_{2}=\frac{2 \pi}{3.540}, \quad L_{3}=\frac{2 \pi}{6.340} .
$$

The wave velocities are

$$
V_{1}=\frac{V_{l}}{0.692}, \quad V_{2}=\frac{V_{l}}{1.18}, \quad V_{3}=\frac{V_{l}}{2.105} .
$$

Figure 4 shows the displacement vector at several points of the section $z=5 \mathrm{~cm}$ for $h=40 \pi$ and for $h=3$, for $t$ an integer.

General conclusions. The solution (24) [or (19), (20)] consists of an infinite number of terms of which all but a finite number (those corresponding to the positive real roots of the frequency equation) decay with $z$.

In some of the following statements we shall consider $\nu=1 / 3$, but the conclusions apply to the general case.

When $\omega$ (frequency) increases, the solution is changed as follows:

1) The number of waves that do not decay with $z$ increases. For example if in the formula $\omega=h V_{\text {l }}$ we take $h$ equal to $1,2,3,3.75, \ldots$ We obtain $1,2,3,4, \ldots$ waves, respectively. For $h=40 \pi$ (ultrasonic frequency) we obtain 130 waves.

2) The rate at which the terms corresponding to the complex roots decay with $z$ increases.

3) Since the wave lengths are given by $2 \pi / h y_{i}$, where $y_{i}$ are the positive roots of Eq. (26) which are in the interval $0<y_{i}<2.5$, as $\omega$ increases the wave length decreases. In our example for $h=40 \pi$, the wave length of the terms of larger amplitude is approximately $1 / 20$, while for $h=3$ it is $2 \pi / 2.076$.

4) The roots of the frequency equation (23) become closer together particularly at $\xi=h(y=1)$ and $\xi=k(y=2)$, and the roots near these two points correspond to waves that travel with velocities that approach $V_{l}$ and $V_{t}$ respectively as $\omega$ increases. The velocity of the wave corresponding to $\xi>k(y>2)$ approaches the velocity of Rayleigh surface wave. In our example for $h=40 \pi$, the terms of larger amplitudes are those with $V \cong V_{l}$.

5) At a section $z=$ constant, the displacement vector will vary in amplitude, direction and sense as $r$ varies. Such variations become larger and faster as $\omega$ increases. (As shown in Fig. 4). 

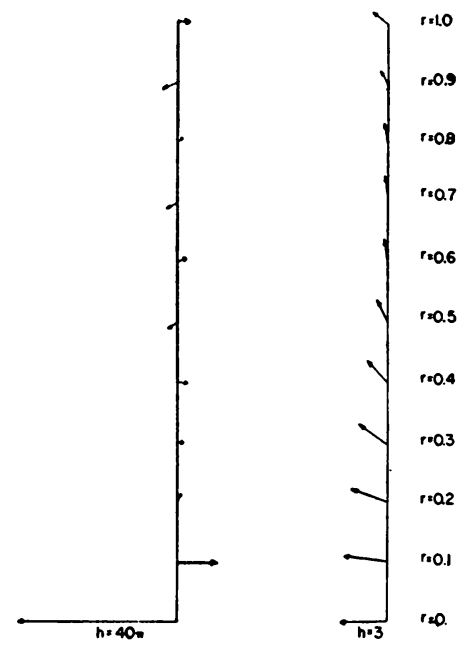

FIg. 4

The amplitude of the wave corresponding to the root $\xi_{i}>k$ decreases when $r$ decreases, and it is practically a surface wave. The amplitudes of the other waves decrease as $V$ increases.

\section{Acknowledgement}

The author wishes to express his gratitude to Professor George F. Carrier for his advice and help in the preparation of this paper. 